\title{
Uniendo investigación, política y práctica educativas: DBR, desafíos y oportunidades
}

Este artículo de fundamentación teórica y reflexión describe la metodología de investigación enfocada al diseño, conocida como Educational Design Research o Designed Based Research (DBR) y señala su carácter participativo, intervencionista y orientado a la resolución de problemas prácticos y al desarrollo de principios y teorías de diseño. Además, propone cuáles son los criterios de calidad aplicables a las investigaciones que siguen esta metodología y sus desafíos. Por último, presenta a la DBR como un instrumento adecuado para establecer puentes transformadores entre la investigación, la práctica y la política educativa.

\section{Palabras clave descriptor}

Investigación participativa, evaluación de la educación, experimento educacional, modelo educacional, indicadores educativos, investigación pedagógica. doi: 10.11144/Javeriana.M7-14. UIPP

Escrito por MARTA ROMERO-ARIZA UNIVERSIDAD DE JAÉN JAÉN, ESPAÑA mromero@ujaen.es

Para citar este artículo / To cite this article / Pour citer cet article / Para citar este artigo Romero-Ariza, M. (2014). Uniendo investigación, política y práctica educativas: DBR, desafíos y oportunidades. magis, Revista Internacional de Investigación en Educación, 7(14), 159-176.

\section{Transferencia a la práctica}

Como refuerzo el argumento esgrimido por Van den Akker (2013), el análisis presentado en este artículo muestra cómo, debido a su carácter participativo, intervencionista, orientado a la práctica y enfocado a la comprensión de procesos y desarrollo de conocimiento científico, la DBR ofrece una oportunidad única para establecer puentes transformadores entre la investigación, la política y la práctica educativa. Este enfoque metodológico acerca la investigación a los problemas y las necesidades de los docentes, con resultados relevantes para la mejora de la enseñanza y la toma de decisiones sobre educación. 


\section{Key words plus}

Participatory Research, Educational

Assessment, Educational Experiment, Educational Model, Educational Indicators, Educational Research.

\section{Abstract}

The study describes and characterizes the research methodology focused on the design known as Educational Design Research or Designed-Based Research (DBR), noting its participatory and interventionist nature, aimed at solving practical problems and developing principles and theories of design. It also discusses which quality criteria apply to investigations that follow this methodology, outlining its challenges. Finally, it presents the DBR as a suitable instrument for establishing transformative bridges between research, practice and education policy.

\section{Transference to practice}

Reinforcing the argument raised by Van den Akker (2013), the analysis presented in this paper shows how, due to its participatory, interventionist and practiceoriented character, as well as its focus on the understanding of processes and the development of scientific knowledge, DBR offers a unique opportunity to bridge research, policy and educational practice. This methodological approach opens a space for having a closer look to the problems and needs of teachers, by providing relevant results for the improvement of both the teaching and decision making processes about education.

\section{Mots clés descripteur}

Recherche participative, évaluation de l'éducation, expérimentation en éducation, modèle d'éducation, indicateurs éducatifs recherche pédagogique.

\section{Résumé}

Ce travail décrit et caractérise la méthodologie de la recherche centrée dans le dessein plus connue comme Educational Design Research o Designed-Based Research (DBR), on souligne son caractère participative, d'intervention orienté à la résolution de problèmes pratiques et au développement de principes et théories de dessein. D'ailleurs, on discute quels sont les critères de qualité qui sont appliqués aux recherches qui suivent cette méthodologie, et qui disposent des défis. Enfin, on présente la DBR en tant qu'un outil adéquat pour établir les ponts transformateurs entre la recherche, la pratique et la politique éducative.

\section{Transfert à la pratique}

En renforçant l'argument présenté par Van den Akker (2013), I'analyse exposée dans cet article montre comment, étant-donnée son caractère participatif, interventionniste, orienté à la pratique et centré dans la compréhension des processus et développements de la connaissance scientifique, la DBR offre une opportunité unique pour établir les ponts transformateurs entre la recherche, la politique et la pratique éducative. Cette perspective méthodologique rapproche la recherche aux problèmes et besoins des enseignants, et elle fournit les résultats importants pour améliorer l'enseignement et la prise des décisions par rapport à l'éducation.
Palavras-chave descritor Pesquisa participativa, avaliação da educação, experimento educacional, modelo educacional, indicadores educativos, pesquisa pedagógica.

\section{Resumo}

Este trabalho descreve e caracteriza a metodologia de pesquisa focada no desenho e conhecida como Educational Design Research ou Designed-Based Research (DBR), destacando seu caráter participativo, intervencionista, orientado à resolução de problemas práticos e ao desenvolvimento de princípios e teorias de desenho. Além do mais, discute que critérios de qualidade são aplicáveis às pesquisas que seguem esta metodologia, esboçando desafios. Por último, apresenta a DBR como um instrumento adequado para estabelecer pontes transformadoras entre a pesquisa, a prática e a política educativa.

\section{Transferência à prática}

Reforçando o argumento esgrimido por Van den Akker (2013), a análise apresentada neste artigo mostra como, devido a seu caráter participativo, intervencionista, orientado à prática e focado ao entendimento de processos e ao desenvolvimento de conhecimento científico, a DBR oferece uma oportunidade única para estabelecer pontes transformadoras entre a pesquisa, a política e a prática educativa. Este enfoque metodológico aproxima a pesquisa dos problemas e necessidades dos docentes, oferecendo resultados relevantes para a melhora do ensino e para as decisões sobre educação. 


\section{Introducción}

Hace ya algunas décadas que diversos autores denuncian la desconexión entre la investigación de los especialistas en el ámbito universitario de la Didáctica y la Pedagogía y los problemas reales, las necesidades e inquietudes que experimentan el profesorado y el alumnado en las aulas (Plomp, 2013). Por ejemplo, el colectivo relacionado con la investigación basada en el diseño o Designed Based Research Collective esgrime que:

[...] la investigación educativa está frecuentemente divorciada de los acontecimientos y problemas asociados a la práctica diaria, lo que genera una brecha de credibilidad y plantea la necesidad de nuevos enfoques de investigación que se dirijan directamente a la práctica y permitan el desarrollo de conocimiento utilizable (The Design Based Research Collective, 2003, p. 5).

Se reivindica un nuevo enfoque metodológico que permita superar esta brecha, es decir, aumentar la relevancia de las investigaciones especializadas, en términos de su utilidad para superar los problemas educativos y mejorar los procesos de enseñanza.

Este es el fundamento de la aproximación conocida en el ámbito anglosajón como Educational Design Research o Design Based Research (DBR). En este trabajo utilizaremos el acrónimo DBR y la traduciremos al castellano como investigación basada en el diseño o, mejor aún, investigación enfocada al diseño.

La DBR promueve la utilización de la investigación sistemática como vía de continua retroalimentación entre teoría y práctica y vincula el desarrollo de conocimiento científico con el diseño de materiales y la resolución eficaz de problemas en el ámbito educativo.

A partir de estas premisas, el propósito de este artículo es introducir esta nueva metodología, para incidir en aquellos aspectos que la justifican y caracterizan y contrastarla con otras metodologías de investigación; además, se indican los desafíos y las oportunidades que este enfoque ofrece.

\section{La investigación enfocada al diseño}

El objetivo de este epígrafe es describir y fundamentar la DBR. Comenzaremos por hablar de su finalidad y mencionaremos distintos trabajos para ilustrar cómo queda reflejada en la formulación de las preguntas de investigación. Luego, discutiremos un modelo genérico que representa, de manera esquemática, las distintas fases que configuran una investigación configurada bajo esta metodología. Por último, analizaremos sus aspectos distintivos y comentaremos similitudes y diferencias con otras metodologías de investigación.

\section{Finalidad de la investigación enfocada al diseño}

La DBR se vincula con dos propósitos fundamentales. Por una parte, pretende dar respuesta a problemas educativos complejos mediante el diseño, el desarrollo y la evaluación de materiales e intervenciones docentes basados en la investigación y, por otra, busca ampliar o validar teorías y principios de diseño que ayuden a entender cómo se aprende y qué procesos apoyan un determinado tipo de aprendizaje, así como cuáles son las características clave de un recurso o una intervención didáctica, responsables de la facilitación o potenciación del aprendizaje.
Descripción del artículo | Article description | Description de l'article Artigo descrição

Este artículo de fundamentación teórica y reflexión llama la atención sobre la conveniencia de aumentar la relevancia de la investigación educativa, en términos de su utilidad para resolver problemas y mejorar los procesos de enseñanza-aprendizaje en las aulas. Para ello, fundamenta y caracteriza un nuevo paradigma de investigación enfocada al diseño, conocido como Educational Design Research o Designed Based Research (DBR). Tras recurrir a publicaciones recientes y a la literatura especializada, se ilustra su carácter participativo, intervencionista, con interés en los procesos y orientado a la práctica y al desarrollo de conocimiento. Por último, se discuten los criterios de calidad y los desafíos que conlleva, así como las oportunidades que ofrece para encontrar sinergias entre la investigación, la política y la práctica educativas. 
No todos los trabajos centrados en el diseño y desarrollo de materiales o intervenciones didácticas pueden considerarse DBR; para que lo sea, es necesario que conceda importancia al avance del conocimiento sobre teorías y principios de diseño y que se apoye en las metodologías de investigación propias de las Ciencias Sociales.

No obstante, para caracterizar la DBR no solo es oportuno enfatizar su finalidad, sino también los rasgos distintivos que están vinculados a su esencia y razón de ser y que se comentarán en apartados posteriores, así como sus similitudes y diferencias con otras aproximaciones como la investigación-acción o el estudio evaluativo de casos.

\section{¿Cómo se formula una pregunta de investigación dentro del enfoque DBR?}

La pregunta que orienta una investigación enfocada al diseño pretende encaminar dicha investigación hacia la consecución de los resultados perseguidos. Evidentemente, la pregunta orientadora se puede formular de muchas maneras, pero con frecuencia la DBR busca identificar las características esenciales de un recurso, una estrategia o una intervención didáctica, responsables de la estimulación del aprendizaje, por lo que suele iniciar con "icuáles son las características de?".

Plomp (2013) menciona distintos trabajos cuya pregunta de investigación sigue ese esquema, por ejemplo, ¿cuáles son las características de la intervención óptima para promover la competencia de escritura de textos académicos en graduados en Educación que van a presentar su trabajo de iniciación a la investigación? (Dowse \& Howie, 2013) o ¿cuáles son las características de los materiales curriculares ideales para promover un trabajo efectivo en el laboratorio de Química, en las escuelas de Tanzania? (Mafumiko, Voogt \& Van den Akker, 2013).

A veces no es posible condensar los condicionantes y requisitos del trabajo en una sola pregunta ni recoger con detalle cuáles son los requerimientos que los interesados demandan del producto final (Gravemeijer \& Cobb, 2013). Aun así, encontramos trabajos que formulan una pregunta de investigación suficientemente específica como para explicitar tanto las características del resultado esperado como los requisitos de partida $y$, de alguna forma, las teorías subyacentes. Este es el caso de la propuesta de Knipples (2002), que se configura en torno a la siguiente pregunta de investigación: ¿Cuál sería la estrategia didáctica adecuada para enseñar genética en los últimos años de educación secundaria, de modo que, teniendo en cuenta las principales dificultades experimentadas por el alumnado en este ámbito, promoviese un aprendizaje significativo y coherente de los fenómenos asociados con la transmisión y la herencia genética? (Knipples, 2002).

Es importante no perder de vista que el objetivo de la DBR, además de resolver problemas o buscar productos o procesos óptimos basados en la investigación, consiste en identificar los rasgos o factores clave y extraer principios de diseño y teorías que relacionen los resultados cognitivos y competenciales deseados con las características de los elementos implicados en la intervención.

Este objetivo dual queda reflejado en algunas preguntas de investigación, como en el caso de aquella que enmarca el estudio de Bielaczyc y Ow (2014): ¿Cómo podemos desarrollar herramientas y prácticas educativas que nos permitan capacitar al alumnado de tercer y cuarto curso de educación primaria para participar en una comunidad que construye conocimiento, así como mejorar nuestra comprensión de los procesos críticos 
para el cambio? La pregunta recoge explícitamente que no solo se buscan herramientas y prácticas que satisfagan una determinada necesidad educativa, sino que también se persigue comprender los procesos, esto es, elaborar teorías explicativas.

\section{El modelo de investigación enfocada al diseño}

De acuerdo con Plomp (2013), son diversas las maneras en las que distintos autores describen la investigación enfocada al diseño, pero existe un consenso generalizado sobre las etapas que esta aproximación conlleva:

\section{Fase 1: investigación preliminar}

Esta primera fase implica el análisis de necesidades y la descripción del problema, así como la revisión de la literatura especializada, con el objeto de identificar trabajos previos con un propósito o énfasis similar, así como establecer la fundamentación y el marco teórico de la investigación.

\section{Fase 2: desarrollo y pilotaje}

Supone la elaboración, revisión y mejora progresiva de prototipos con base en estudios sistemáticos tras sucesivos ciclos de investigación. El elemento clave que orienta la mejora es la evaluación formativa resultante de cada iteración.

Fase 3: evaluación final

Su principal finalidad es valorar si la intervención o el producto final satisface los objetivos y requerimientos planteados en principio. Puesto que esta etapa suele integrar recomendaciones para la mejora, algunos autores la denominan evaluación semisumativa (Plomp, 2013). Esta fase incluye el análisis y la reflexión sistemática destinada a la obtención de conclusiones que orienten futuros diseños.

La tabla 1 muestra la identificación de las fases asociadas con una investigación enfocada al diseño y la terminología utilizada por varios autores:

Tabla 1.

Fases de una investigación enfocada al diseño

\begin{tabular}{llll}
\hline & Fase 1 & Fase 2 & Fase 3 \\
\hline $\begin{array}{l}\text { Cobb, Confrey, DiSessa, } \\
\text { Lehrer y Schauble (2003) }\end{array}$ & $\begin{array}{l}\text { Preparación del experimento } \\
\text { sobre diseño }\end{array}$ & $\begin{array}{l}\text { Realización del experimento } \\
\text { sobre diseño }\end{array}$ & Análisis retrospectivo \\
\hline Reeves (2000; 2006) & $\begin{array}{l}\text { Análisis del problema } \\
\text { Desarrollo de prototipos ba- } \\
\text { sados en la literatura }\end{array}$ & $\begin{array}{l}\text { Ciclos iterativos de refinado prototipos mediante in- } \\
\text { vestigación }\end{array}$ & $\begin{array}{l}\text { Reflexión para generar prin- } \\
\text { cipos de diseño y mejorar la } \\
\text { resón de problemas en } \\
\text { la práctica }\end{array}$ \\
\hline McKenney (2001) & $\begin{array}{l}\text { Análisis de necesidades y del } \\
\text { contexto }\end{array}$ & $\begin{array}{l}\text { Diseño, desarrollo y evalua- } \\
\text { ción formativa de sucesivos } \\
\text { prototipos }\end{array}$ & Evaluación semisumativa \\
\hline \hline
\end{tabular}

Fuente: elaboración propia

Según esta visión lineal, el desarrollo de principios y teorías de diseño se vincula con la fase final. No obstante, algunos autores proponen un modelo en el que la construcción de conocimiento científico se integra de forma transversal. En esta línea, Wademan (2005) considera que la mejora progresiva de prototipos en ciclos sucesivos de refinado va acompañada de una aproximación gradual hacia teorías y principios de 
diseño. Esto es, el desarrollo de productos, entendidos como materiales docentes o intervenciones didácticas, tiene lugar de forma paralela al avance del conocimiento.

La figura 1 representa un modelo genérico de investigación enfocada al diseño. El diagrama incluye componentes presentes en los modelos propuestos por distintos autores (McKenney, 2001; Plomp, 2013; Reeves, 2000; 2006; Wademan, 2005).

Figura 1

Modelo genérico de investigación enfocada al diseño

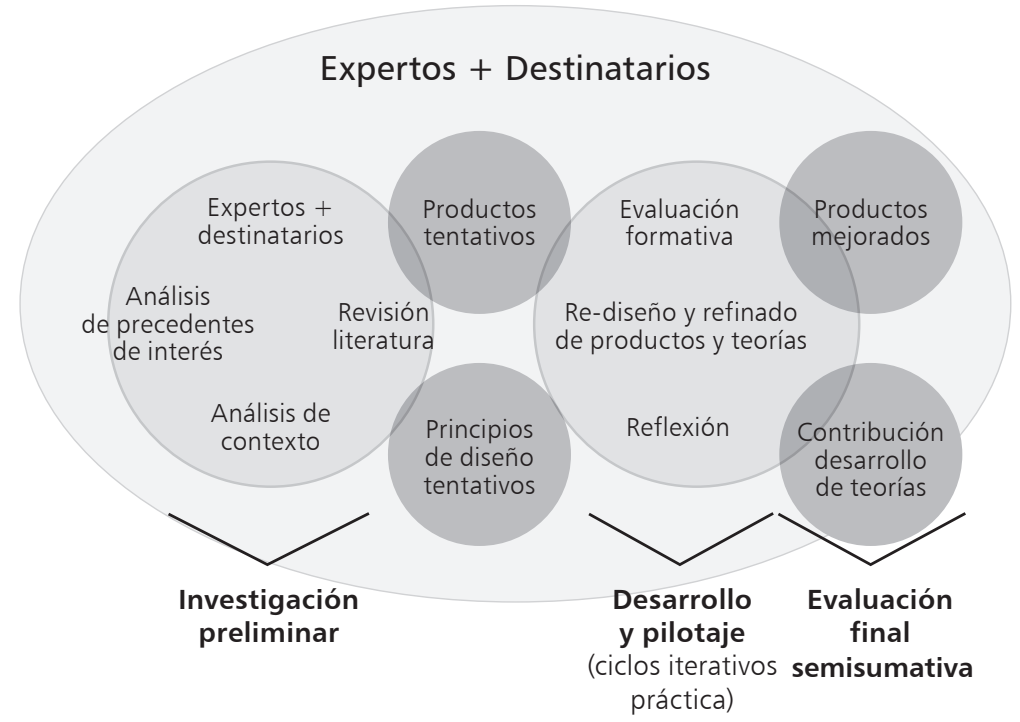

Aproximación progresiva a principios de diseño, recursos y estrategias mejoradas

Fuente: elaboración propia

En la parte inferior de la figura se delimitan las tres fases de la DBR (investigación preliminar, desarrollo y pilotaje y evaluación final semisumativa). En el espacio asociado con cada fase se representa la interacción entre los principales agentes y elementos implicados en ella.

La figura 1 muestra cómo la investigación preliminar implica la colaboración de investigadores y profesorado (expertos y destinatarios) para identificar necesidades, definir el problema y analizar en conjunto el contexto de trabajo. Asimismo, supone la revisión de la bibliografía especializada, para definir el marco teórico e identificar precedentes de interés. La investigación preliminar permite definir unos prototipos iniciales (o productos tentativos) e identificar principios de diseño presentes en la literatura, que serán validados o refinados mediante la investigación posterior.

La etapa de desarrollo y pilotaje implica ciclos iterativos de aplicación, evaluación y mejora progresiva de los productos. Cada ciclo conlleva una microinvestigación enfocada a analizar la relación entre las características de los recursos y las estrategias que se utilizaron y su influencia en los procesos de enseñanza-aprendizaje. Esta investigación permite orientar el cambio hacia la mejora. Los ciclos sucesivos de refinado originan productos mejorados y contribuyen a la construcción de conocimiento en la forma de principios de diseño y teorías. 
Por último, el objetivo de la etapa final es valorar hasta qué punto los productos finales (intervenciones, recursos o estrategias didácticas) satisfacen los propósitos iniciales o permiten resolver el problema planteado en un principio.

En la parte superior de la figura se resalta el carácter participativo de la DBR señalado por diversos autores, que definen como rasgos distintivos de esta aproximación la colaboración entre expertos y destinatarios (Kelly, 2006; Nieveen, 1999; Plomp, 2013; Van den Akker, 1999). Esto supone la implicación del profesorado en el análisis del problema y el contexto en el que se desarrolla, así como la colaboración en el análisis de situaciones y en la búsqueda de soluciones basadas en la investigación.

La figura 2 representa cómo el número de iteraciones depende, entre otras cosas, de la evaluación formativa llevada a cabo en cada ciclo. Si dicha evaluación revela que se han alcanzado los objetivos perseguidos, la investigación podría darse por finalizada. Si no es así, se muestra la necesidad de abordar un nuevo proceso de refinado que permita aproximarse a los productos buscados.

Figura 2.

Papel de los ciclos de refinado en la mejora progresiva de prototipos hasta alcanzar los objetivos perseguidos en una investigación enfocada al diseño

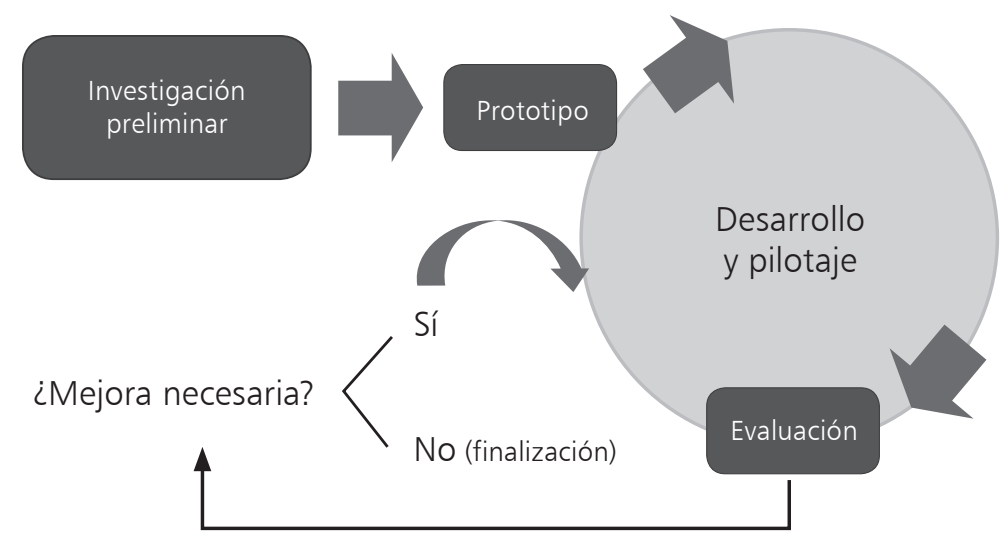

Fuente: elaboración propia con base en Plomp (2013, p. 17)

De acuerdo con Cobb, Confrey, DiSessa, Lehrer y Schauble (2003), las investigaciones enfocadas al diseño conllevan tanto la "ingeniería" de determinadas formas de aprendizaje como el estudio sistemático de esas formas de aprender en el contexto predefinido para fomentarlas. El contexto de estudio se somete a análisis y revisión y los ciclos sucesivos de refinado fundados en la evaluación formativa de los mismos desempeñan un papel similar a las variaciones sistemáticas de variables llevadas a cabo en un experimento.

\section{Características de la investigación enfocada al diseño}

En la descripción del modelo de DBR comentada en el apartado anterior se han mostrado, de manera más o menos explícita, algunos de los rasgos distintivos de la investigación enfocada al diseño. En esta sección vamos a enfatizar los más destacados. 
Además de la naturaleza participativa y colaborativa asociada con la implicación de los destinatarios en el proceso DBR, existe un consenso generalizado sobre las cualidades que exhibe (Plomp, 2013; Van den Akker, Gravemeijer, McKenney \& Nieveen, 2006):

\section{Carácter intervencionista}

Esta cualidad se debe a que el principal propósito de la DBR es el diseño de intervenciones en contextos reales, para mejorar la práctica educativa o resolver problemas.

\section{Carácter iterativo}

La investigación incorpora ciclos sucesivos de análisis, diseño, desarrollo, evaluación y revisión.

\section{Énfasis en el estudio y la comprensión de los procesos}

La investigación enfocada al diseño trata de evitar un modelo de intervención en la práctica basado en el ajuste ciego de condiciones-consecuencias. Por lo contrario, busca la comprensión de los procesos, con el objeto de disponer de criterios y principios para el diseño y la toma de decisiones.

\section{Orientación hacia la utilidad práctica}

La DBR procura desarrollar recursos y estrategias compatibles con las necesidades y circunstancias de los destinatarios y que, por tanto, sean útiles para la resolución de problemas y la mejora de la práctica en el aula.

\section{Orientación al desarrollo de teorías}

Es importante tener en cuenta que la investigación enfocada al diseño sigue una aproximación holística que no enfatiza en el estudio de variables aisladas. Van den Akker, Gravemeijer, McKenney y Nieveen (2006) señalan que, aunque los investigadores en la DBR se centran en objetos y procesos concretos (intervenciones) en un contexto determinado, se aproximan a ellos con el propósito de comprenderlos como parte de un fenómeno complejo. La importancia que la DBR concede a las circunstancias en las que tienen lugar los procesos de enseñanza aprendizaje explica por qué esta aproximación no busca la generalización extensible a cualquier contexto. En contraste con las generalizaciones estadísticas que pretenden extrapolar las características de una muestra a una población entera, la DBR persigue una generalización analítica. Este aspecto se discutirá con mayor detalle en apartados posteriores.

\section{Similitudes y diferencias entre enfoques de investigación relacionados con la DBR}

La metodología de investigación enfocada al diseño está relacionada con diversas aproximaciones que comparten objetivos comunes: diseñar y evaluar intervenciones y recursos didácticos basados en la investigación y construir conocimiento al respecto, en forma de teorías sobre aprendizaje y principios de diseño.

Entre dichas aproximaciones se incluyen los trabajos de desarrollo y diseño curricular, los estudios evaluativos de caso, la investigación-acción y los estudios de validación de teorías y principios de diseño. A continuación se comentan puntos de confluencia y diferencia entre estas metodologías investigativas.

Los estudios de desarrollo curricular procuran la obtención de materiales, recursos, estrategias e intervenciones didácticas, mientras los estudios evaluativos de caso pretenden valorar la adecuación de estas propuestas, en forma de programas de acción en Ciencias Sociales, en atención a los objetivos propuestos para dichos programas. Ambas aproximaciones comparten con la DBR el objetivo de contribuir a la generación o a la evaluación de propuestas curriculares. No obstante, pueden no implicar el planteamiento de principios o teorías de diseño.

A semejanza de la DBR, la investigación-acción utiliza el estudio cualitativo y la reflexión sistemática sobre la práctica en el aula como principales herramientas de mejora e innovación educativa. Además, comparte con ella su carácter intervencionista y participativo, pero no necesariamente el interés en el desarrollo de principios y teorías de diseño.

En oposición al resto de enfoques comentados y en común con la DBR, los estudios de validación de teorías propenden por la generalización y la construcción de conocimiento que permita orientar futuros diseños e intervenciones. Para ello, se centran en el desarrollo o la validación de teorías sobre aprendizaje que expliquen cómo las cualidades del aprendizaje adquirido se ven influenciadas por el entorno, los recursos, las estrategias y las condiciones en las que dicho aprendizaje tiene lugar.

Al margen de lo comentado en relación con los trabajos de diseño y desarrollo curricular, Van den Akker (1999; 2013) propone un modelo de desarrollo curricular basado en la investigación, que puede considerarse como un espécimen dentro de la familia DBR. El autor recalca la importancia de recurrir a la investigación previa para establecer criterios de calidad y métodos adecuados y reivindica la contribución de tal desarrollo curricular tanto a la obtención de intervenciones educativas optimizadas como a la propuesta de principios de diseño. 
En la misma línea, los estudios de planteamiento o validación de teorías centrados en la investigación de entornos y estrategias de aprendizaje pueden ser considerados como especímenes de una misma familia, la DBR (Plomp, 2013; Van den Akker, 2013).

\section{Criterios de calidad asociados con la DBR}

En esta sección vamos a analizar qué criterios se consideran asociados con un buen trabajo dentro de la metodología DBR. Dichos criterios son coherentes con la finalidad y los rasgos característicos de la investigación enfocada al diseño.

Plomp (2013) cita a Nieveen (1999) para destacar tres criterios de calidad: validez, utilidad o carácter práctico y efectividad. La validez de una investigación depende del rigor científico y de la consistencia y fiabilidad de los instrumentos utilizados. Nieveen (1999) distingue entre validez de contenido y validez de constructo. El primer término hace referencia a la fundamentación teórica, que implica una revisión del estado de la cuestión en el ámbito de conocimiento en el que se enmarca dicho trabajo con un análisis crítico de la literatura especializada y los antecedentes relevantes. El segundo término se refiere a la validez de los instrumentos y métodos empleados, esto es, a la necesaria consistencia de los elementos implicados en la investigación.

El segundo criterio de calidad es la utilidad o el carácter práctico de la investigación y de sus resultados. De acuerdo con los principales propósitos de la DBR, un trabajo enmarcado en este enfoque es de calidad si origina productos considerados de utilidad por el profesorado o los usuarios finales a quienes va dirigida la investigación. También es importante que los potenciales interesados encuentren el resultado práctico y aplicable en el contexto y las circunstancias en las que desarrollan su práctica profesional y que dicha aplicación es coherente y compatible con las intenciones de los investigadores.

Por último, otro indicio de calidad del diseño basado en la investigación es la efectividad, consistente en que su resultado evidencia la consecución de los objetivos planteados en principio.

No obstante, en este punto es conveniente llamar la atención sobre dos aspectos:

1. La importancia de diferenciar entre las características esperadas y las reales.

2. La relación jerárquica existente entre los cuatro criterios de calidad mencionados.

La primera matización hace referencia a la distinción entre las validaciones por expertos de un instrumento o de un producto y la evaluación de la utilidad o eficiencia de estrategias o recursos, que queda demostrada una vez que se implementan en la práctica educativa (Nieveen \& Folmer, 2013).

El segundo aspecto destacado alude a la importancia relativa concedida a cada uno de los criterios de calidad, los cuales siguen una jerarquía lógica. Para ilustrar este aspecto, Plomp (2013) cuestiona si un recurso o una estrategia didáctica no se puede utilizar o aplicar en la práctica diaria, ¿qué sentido tiene estudiar su efectividad? $O$, si un instrumento o un recurso no está diseñado de forma coherente o consistente, imerece la pena investigar su carácter práctico o su efectividad? 
Asimismo, es necesario hacer notar que no todos los criterios de calidad cobran igual valor a lo largo de una investigación enfocada al diseño, pues según la fase, predominan unos u otros: en la fase de investigación preliminar, el énfasis reside en la relevancia o validez de contenido; en la fase de desarrollo de prototipos, se presta especial atención a la consistencia o validez de constructos y en la fase final, predominan los criterios de calidad asociados con la utilidad y la efectividad de los productos desarrollados.

\section{Desafíos asociados con la investigación enfocada al diseño}

Este apartado abre el camino a la discusión de dos temas críticos de interés relacionados con la DBR: por un lado, pone sobre la mesa las dificultades derivadas de la investigación de contexto reales y del carácter intervencionista y participativo de este tipo de trabajos; por otro, discute aspectos relacionados con el rigor científico, la validez y la generalizabilidad.

\section{Desafíos derivados del estudio intervencionista y participativo de contextos reales}

La DBR surge como respuesta a las críticas que denuncian la falta de relevancia y utilidad para la práctica educativa de gran parte de las investigaciones en Didáctica. Para enfrentar esta reivindicación, la DBR parte de los problemas y las necesidades de los profesionales en el aula y busca soluciones basadas en la investigación, en colaboración con los implicados en dichas problemáticas.

No obstante, este enfoque conlleva una serie de dificultades o desafíos. Vamos a comentar los referentes a la intervención de los distintos participantes, el papel del investigador y la necesidad de extraer suficiente información para facilitar la comprensión de fenómenos complejos y el desarrollo de principios y teorías de diseño.

La implicación de colectivos de interés en la investigación (profesorado, estudiantes, autoridades educativas) supone que, a veces, se encuentra una cierta reticencia de estos individuos a participar o, incluso, un conflicto de intereses, lo que dificulta la colaboración abierta y sincera. En esta línea, Plomp (2013) cita a McKenney, Nieveen, y Van den Akker (2006) para llamar la atención sobre la importancia de trabajar una atmósfera sólida de colaboración entre investigadores y el resto de participantes, basada en la confianza y el beneficio mutuo. Esta colaboración es esencial para construir una visión rica de los procesos de enseñanza aprendizaje y las complejas relaciones entre distintos factores, así como para garantizar la utilidad práctica del estudio.

En este tipo de trabajos, el investigador suele desempeñar también el rol de diseñador, evaluador y asesor. Varios autores aportan recomendaciones para afrontar los posibles inconvenientes derivados de ello (McKenney, Nieveen y Van den Akker 2006; Plomp, 2013; Van den Akker, 2005):

- Estar preparado para asumir los roles como diseñador y asesor sin perder de vista el papel como investigador.

- Mantenerse receptivo hacia posibles cambios en el diseño de investigación que puedan precisarse en el trascurso de la misma.

- Estar abierto a las sugerencias y los intereses expresados por los participantes a lo largo de lo que suele ser una larga colaboración. 
- Conjugar la receptividad y capacidad de adaptación con la necesidad de garantizar la robustez del diseño y el desarrollo de la investigación.

- Recurrir a evaluadores externos o critical friends.

Para ello, el investigador no solo requiere habilidades de comunicación y empatía, sino también un conocimiento sólido de las metodologías de investigación y los criterios de rigor científico, que le permitan afrontar los cambios pertinentes en el diseño inicial del trabajo, maximicen su calidad científica y minimicen las posibles amenazas que dichos cambios pudieran entrañar (McKenney et al., 2006).

\section{Desafíos asociados con la validez y la fiabilidad de las investigaciones DBR}

Debido al énfasis en la comprensión profunda de complejos fenómenos e interrelaciones que tienen lugar en los contextos reales de enseñanza aprendizaje, esta aproximación recurre con frecuencia al estudio y análisis cualitativo de datos, con el objeto de proponer principios y teorías de diseño para orientar la práctica educativa.

Sin embargo, para asegurar el interés práctico y la efectividad real de los productos derivados de la DBR (recursos, estrategias y entornos de enseñanza-aprendizaje), varios autores reivindican la importancia de completar las investigaciones centradas en el diseño con estudios de campo que validen la utilidad y eficiencia de estos productos. Es por eso que también se recurre a análisis cuantitativos.

Los ambiciosos objetivos de la DBR exigen frecuentemente investigaciones complejas y de larga duración que han de cumplir con los requerimientos de rigor científico asociados tanto a las investigaciones de corte cuantitativo, como sobre todo las de corte cualitativo.

Para garantizar dicho rigor McKenney y otros autores (2006) y Plomp (2013) recomiendan:

- Consolidar un marco conceptual explícito basado en la revisión de la literatura especializada, en el análisis de estudios precedentes de interés o en el asesoramiento de expertos.

- Establecer un diseño de investigación congruente. Para ello, algunos autores coligan estas cualidades con la definición de una sólida cadena de razonamiento, lo que implica que cada una de las partes de la investigación sea consistente (Krathwohl, 1998; Plomp, 2013).

- Recurrir a la triangulación mediante el uso de diferentes fuentes, tipos de datos, procedimientos, evaluadores y teorías, para contrastar los resultados, evitar sesgos y garantizar la fiabilidad.

- En relación con el punto anterior, se recomienda aplicar una amplia variedad de métodos y tácticas, por ejemplo, invitar a profesorado externo al proyecto, a autoridades educativas o a otros investigadores como critical friends.

- Usar descripciones ricas y completas del contexto en donde se adelanta la investigación, integrar distintas perspectivas y justificar con detalle las decisiones de diseño que se toman y la interpretación de datos y resultados.

- Contrastar las descripciones e interpretaciones de los investigadores con las fuentes originales para aumentar su validez interna. 
- Prestar atención a la validez y fiabilidad de los instrumentos y métodos utilizados, de acuerdo con los criterios de rigor científico para la investigación en Ciencias Sociales.

\section{Generalizabilidad de las investigaciones DBR}

Como se ha expuesto en apartados anteriores, la DBR pretende aportar soluciones basadas en la investigación de problemas educativos complejos y mejorar y optimizar los procesos de enseñanza aprendizaje. Para ello, no busca un modelo de ajustes ciego entre las condiciones de trabajo y los resultados obtenidos, sino que, por lo contrario, apunta a comprender-explicar los mecanismos que justifican las interrelaciones entre los factores implicados en un proceso formativo. De acuerdo con Cobb y otros autores (2003), este tipo de estudios aporta mayor entendimiento de una determinada "ecología de aprendizaje", interpretada como un sistema complejo de múltiples elementos de diferentes tipos. Este conocimiento permite establecer un diseño con estos elementos y anticipar cómo esta configuración influirá en el aprendizaje.

Dicho conocimiento se puede expresar en la forma de principios de diseño o teorías "locales", pero, ¿son los productos de la DBR generalizables fuera del contexto de estudio en donde han sido desarrollados? Edelson (2006) enfatiza la importancia de que la DBR dé lugar a teorías generalizables.

La visión de procesos y entornos educativos como "ecologías de aprendizaje" reconoce la influencia que estos tienen sobre el aprendizaje adquirido y, por ello, concede especial valor al análisis y a la comprensión de tal ecología. Desde este punto de vista, podría pensarse que no tiene sentido hacer generalizaciones independientes del contexto, puesto que este se considera un factor determinante.

Además, la DBR comparte con las metodologías de investigación cualitativas el interés por desarrollar visiones profundas y holísticas de los fenómenos, con el fin de interpretar y explicar sistemas complejos y aportar información suficiente que oriente los procesos de diseño y mejora (evaluación formativa). Por ello, no tiene como principal propósito la generalización estadística del objeto de estudio a la población completa. En estudios cuantitativos, la fiabilidad y generalizabilidad de un estudio, avaladas por pruebas estadísticas, aportan una medida objetiva de la estabilidad de las medidas o los resultados obtenidos frente a variaciones aleatorias. Entonces, ¿qué tipo de generalización predomina en el DBR?

El enfoque DBR reivindica una generalización analítica, es decir, basada en la descripción detallada y la comprensión profunda de las complejas interrelaciones observadas entre los distintos componentes que facilitan o dificultan un determinado aprendizaje, lo que permite extraer principios de diseño y teorías locales.

Según Van den Akker (1999), existen dos clases de principios de diseño: los procesuales y los sustantivos. Los primeros hacen referencia a las relaciones observadas entre los tipos de actividades y los procesos que se han puesto en juego en una determinada intervención educativa y los resultados obtenidos. Los principios sustantivos aluden a las características de los recursos y las estrategias utilizadas y la influencia que estos tienen sobre el aprendizaje.

En referencia a Van den Akker (1999), Plomp (2013) señala que dichos principios son heurísticos y, en principio, pueden ser tomados como recomendaciones basadas en la experiencia para hacer frente a situaciones o problemas particulares. Sin embargo, debido a su carácter heurístico, 
han sido extraídos de un contexto preciso y no hay garantía de éxito en otros escenarios. Estos principios ganarán potencial cuando se validen en varios entornos.

En esta línea, Yin (2003) indica que los resultados de los estudios de caso no se pueden generalizar de manera automática a un entorno más amplio, aunque el investigador intente que el conocimiento derivado de su estudio pueda integrar una teoría explicativa con un alcance superior al del caso estudiado. Por ello, reivindica que los principios de diseño y las teorías locales deberían probarse al replicar el estudio, con objeto de contrastar los hallazgos. Una vez que se hayan llevado a cabo varias replicaciones, los principios de diseño o de teorías locales podrían ser aceptados para contextos similares. La generalización derivada de este reducido número de replicaciones es la misma que la utilizada por los científicos en un laboratorio cuando consideran extrapolables (bajo las mismas condiciones) los resultados obtenidos en un determinado experimento (Yin, 2003).

En este punto es esencial llamar la atención sobre el hecho de que, aunque los principios de diseño y las teorías locales se pueden reforzar con replicaciones en contextos similares, en investigación en Ciencias Sociales cada entorno tiene sus características peculiares, lo que justifica que los principios de diseño deban ser tomados como meras recomendaciones basadas en evidencias prácticas, pero nunca como certezas. Varios autores (Plomp, 2013; Reeves, 2006) citan la afirmación de Lee Cronbach, uno de los investigadores en Educación más influyentes del siglo XX: "Cuando se concede suficiente peso específico a las condiciones locales, cada generalización es una hipótesis de trabajo, no una conclusión" (Cronbach, 1975, p. 125).

\section{Algunos trabajos recientes de investigación enfocada al diseño}

A continuación se presentan dos trabajos de reciente publicación, que ilustran cómo esta metodología va cobrando importancia en la investigación en Ciencias Sociales, en particular en Educación. El primero ofrece un ejemplo de estudio enfocado a la validación de teorías dentro de la DBR y el segundo sitúa el énfasis en el diseño y desarrollo de prácticas y herramientas óptimas para alcanzar una determinada finalidad formativa.

Como ejemplo de validación de teorías, comentamos el estudio adelantado por Zuiker y Whitaker (2014). Estos autores diseñaron una investigación encaminada a valorar la adecuación de un nuevo modelo didáctico que denominan $5 \mathrm{E}+\mathrm{l} / \mathrm{A}$, fundamentado en modelos precursores. En primer lugar, se hizo una investigación preliminar y se recurrió a la literatura especializada para revisar la evolución conceptual de los modelos educativos basados en la secuencia de las 5E; asimismo, se analizaron las evidencias experimentales sobre el impacto positivo de cada uno de ellos (Bybee et al., 2006; Duran, Duran, Haney \& Scheuermann, 2011; Eisenkraft, 2003; Fletcher, 2011; Siribuannam \& Tayraukham, 2009).

El prototipo de modelo $5 \mathrm{E}+\mathrm{l} / \mathrm{A}$ se implementó en el aula y se sometió a estudio y valoración por medio de ciclos de análisis cualitativo. Para su evaluación se utilizaron varias fuentes de datos (grabaciones en audio y video de clases diseñadas de acuerdo con este modelo educativo, observaciones participantes, entrevistas abiertas y semiestructuradas, etc.). En principio, las categorías se establecieron de forma deductiva a partir del modelo. El primer análisis reflejó la necesidad de proponer y refinar nuevas categorías de modo inductivo, hasta que sucesivas revisiones condujeron a una interpretación congruente y sólida de los datos. La evaluación formativa integrada de manera transversal al proceso, permitió adaptar progresivamente el modelo y optimizar la intervención educativa fundada en él. Los resultados mostraron la adecuación del modelo para promover un aprendizaje significativo del concepto de densidad en una escuela elemental de Estados Unidos.

En la línea discutida en apartados previos de este artículo, Zuiker y Whitaker (2014) mencionan la generalizabilidad analítica y ecológica de este tipo de trabajos y señalan que sería interesante hacer replicaciones en contextos similares o diferentes y plantear futuros estudios de campo cuasi experimentales que refuercen la validez del modelo.

El otro trabajo que se analiza en este artículo es el publicado por Juuti y Lavonen (2013). Estos autores utilizaron un enfoque DBR enfocado al diseño de un entorno virtual de aprendizaje para la enseñanza de la Física en educación primaria.

En la investigación preliminar, la revisión de la literatura permitió fundamentar una metodología de trabajo basada en la colaboración entre investigadores y profesores para el diseño y la evaluación de recursos e intervenciones educativas. Al mismo tiempo, se delimitó un marco teórico que incluía aspectos psicológicos y epistemológicos asociados con el tipo de aprendizaje de la Física que se pretendía conseguir en el alumnado de primaria y cómo promoverlo. Por último, se revisaron trabajos sobre diseño y uso efectivo de recursos didácticos tecnológicos.

Con el fin de conocer la visión del profesorado, sus expectativas y necesidades, se solicitó a catorce participantes de un curso de desarrollo profesional que reflejaran por escrito cómo se veían a sí mismos en su función de docentes de Física, cómo aprendían sus estudiantes y cuáles eran sus necesidades para enseñar Física en educación primaria. Tres de los profesores 
fueron seleccionados para profundizar en sus visiones mediante entrevistas semiestructuradas.

A partir de esta información se determinó que el entorno de enseñanza de la Física tendría que exhibir las siguientes características:

- Ofrecer contenidos actualizados, tener en cuenta las últimas tendencias pedagógicas y presentar una estructura organizativa adecuada.

- Los contenidos habrían de ser concretos y permitir la conexión con las experiencias y el contexto próximo del alumnado.

- El entorno de aprendizaje tendría que promover el aprendizaje activo, ser fácil de usar y permitir el planteamiento de modelos cualitativos para explicar fenómenos físicos.

- La herramienta debería facilitar el aprendizaje conceptual de modelos básicos de mecánica newtoniana.

- Sería deseable que permitiera la comunicación con expertos y la interacción entre compañeros de clase.

Una vez finalizada la investigación preliminar y establecidos los objetivos del trabajo, se abordó la fase de desarrollo, pilotaje y mejora sucesiva de prototipos en ciclos iterativos de aplicación, evaluación formativa y refinado de la herramienta. La tabla 2 resume las fases de la investigación.

Tabla 2.

Investigación enfocada al diseño de Juuti y Lavonen (2013)

\begin{tabular}{|c|c|c|c|c|}
\hline \multicolumn{2}{|c|}{ Fase } & Pregunta de investigación & Recogida de datos & Resultados \\
\hline \multirow[t]{2}{*}{$\begin{array}{l}\text { Investigación } \\
\text { preliminar }\end{array}$} & $\begin{array}{l}\text { Revisión de la } \\
\text { literatura }\end{array}$ & $\begin{array}{l}\text { ¿Qué podemos aprender } \\
\text { de investigaciones y mar- } \\
\text { cos conceptuales previos? }\end{array}$ & & \\
\hline & $\begin{array}{l}\text { Análisis de nece- } \\
\text { sidades }\end{array}$ & $\begin{array}{l}\text { ¿Qué necesita el profe- } \\
\text { sorado de primaria en } \\
\text { un entorno virtual para } \\
\text { enseñar Física? }\end{array}$ & $\begin{array}{l}\text { Catorce informes es- } \\
\text { critos y tres entrevistas } \\
\text { semiestructuradas }\end{array}$ & $\begin{array}{l}\text { Características de cali- } \\
\text { dad requeridas para el } \\
\text { producto final }\end{array}$ \\
\hline \multirow[t]{8}{*}{$\begin{array}{l}\text { Desarrollo y } \\
\text { pilotaje }\end{array}$} & \multirow[t]{2}{*}{$\begin{array}{l}\text { Ciclo 1: un } \\
\text { grupo clase, dos } \\
\text { lecciones, un } \\
\text { tópico }\end{array}$} & \multirow[t]{2}{*}{$\begin{array}{l}\text { ¿Cuáles son los principa- } \\
\text { les problemas que presen- } \\
\text { ta el prototipo } 1 \text { ? }\end{array}$} & \multirow[t]{2}{*}{$\begin{array}{l}\text { Grabaciones en video, } \\
\text { observación participan- } \\
\text { te, discusión reflexiva } \\
\text { posterior investigador- } \\
\text { profesor }\end{array}$} & $\begin{array}{l}\text { Conexión insuficiente } \\
\text { entre el modelo físico y } \\
\text { las observaciones y ex- } \\
\text { periencias el alumnado. }\end{array}$ \\
\hline & & & & $\begin{array}{l}\text { El profesor ha de des- } \\
\text { empeñar un papel clave }\end{array}$ \\
\hline & \multirow{3}{*}{$\begin{array}{l}\text { Ciclo 2: un } \\
\text { grupo clase, } \\
\text { ocho lecciones } \\
\text { dobles, todos los } \\
\text { tópicos }\end{array}$} & \multirow{3}{*}{$\begin{array}{l}\text { ¿Cómo interaccionan y } \\
\text { aprenden los estudiantes } \\
\text { con el recurso? } \\
\text { ¿Les gusta? }\end{array}$} & $\begin{array}{l}\text { Grabaciones en video, } \\
\text { observaciones }\end{array}$ & $\begin{array}{l}\text { Encuentran la herra- } \\
\text { mienta amena }\end{array}$ \\
\hline & & & $\begin{array}{l}\text { Cuestionario a estu- } \\
\text { diantes }\end{array}$ & $\begin{array}{l}\text { Aprecian las historias } \\
\text { narradas }\end{array}$ \\
\hline & & & $\begin{array}{l}\text { Entrevistas a dos pares } \\
\text { de estudiantes }\end{array}$ & $\begin{array}{l}\text { Relacionan la Física solo } \\
\text { con la escuela }\end{array}$ \\
\hline & \multirow{3}{*}{$\begin{array}{l}\text { Ciclo 3: tres } \\
\text { grupos clase } \\
\text { de distintas } \\
\text { escuelas, tres } \\
\text { lecciones dobles, } \\
\text { tres profesores y } \\
77 \text { estudiantes }\end{array}$} & \multirow{3}{*}{$\begin{array}{l}\text { ¿Hasta qué punto los } \\
\text { alumnos han aprendido } \\
\text { Física clásica? } \\
\text { ¿Hay diferencias de } \\
\text { género? }\end{array}$} & $\begin{array}{l}\text { Grabaciones de video, } \\
\text { observaciones }\end{array}$ & $\begin{array}{l}\text { Pequeño progreso } \\
\text { conceptual }\end{array}$ \\
\hline & & & \multirow{2}{*}{$\begin{array}{l}\text { Test estandarizado de } \\
\text { comprensión conceptual }\end{array}$} & Diferencias entre clases \\
\hline & & & & $\begin{array}{l}\text { Mejores resultados } \\
\text { asociados con las clases } \\
\text { que utilizaron narrativa } \\
\text { de historias }\end{array}$ \\
\hline $\begin{array}{l}\text { Evaluación } \\
\text { semisumativa }\end{array}$ & \multicolumn{4}{|c|}{$\begin{array}{l}\text { Entorno web de mejorada usabilidad, con trayectorias de aprendizaje para una mayor guía. Importan- } \\
\text { cia concedida al uso de historias para ilustrar los tópicos y a la conexión con el entorno próximo del } \\
\text { alumnado. Impacto bajo en el desarrollo de conceptos de Física newtoniana. }\end{array}$} \\
\hline
\end{tabular}

Fuente: elaboración propia basada en Juuti y Lavonen (2013) 
La tabla muestra el papel clave del profesorado en el proceso y el carácter participativo, orientado a la práctica de la investigación.

Como resultado del análisis y de la evaluación formativa, el entorno web refinó progresivamente su diseño para mejorar su usabilidad, ofrecer al profesorado más oportunidades de conectar lo enseñado con el entorno próximo del alumnado y enfatizar el uso de las historias narradas para trabajar los tópicos de Física.

\section{La DBR como puente entre la investigación, la política educativa y el aula: en busca de sinergias}

En principio podría pensarse que el objetivo último de la investigación educativa debería ser contribuir a la mejora de los procesos de enseñanza aprendizaje en contextos reales. No obstante, desde diversos lugares del mundo se denuncia que los resultados de la investigación en Didáctica tienen un escaso impacto en las aulas. Esto lleva a inferir que dichos trabajos tienen poco interés y escasa relevancia para los docentes y, por lo tanto, bajo potencial para mejorar la práctica diaria.

La DBR recoge esta reivindicación y convierte en su principal foco de interés al desarrollo de conocimiento científico para resolver problemas educativos en contextos reales.

Mas ¿qué hace a la DBR una metodología más próxima a este objetivo que el resto de enfoques de investigación? La respuesta puede encontrarse en su carácter intervencionista y dirigido a la mejora de la práctica, en la importancia concedida a la aplicabilidad, utilidad y eficiencia de los productos elaborados y en su naturaleza participativa. En este trabajo se ha mostrado cómo la DBR concede especial importancia a la implicación en el estudio de los destinatarios y otros colectivos de interés (profesorado, alumnado, autoridades educativas), lo que permite acercar la investigación educativa a sus intereses y necesidades.

Por otro lado, Van den Akker (2013) señala que es realista reconocer que el diseño y el desarrollo curricular en el mundo entero destacan por su débil relación con la investigación educativa, pues la toma de decisiones en este ámbito está influenciada por factores sociales y políticos, los proyectos curriculares rara vez surgen o atienden al seguimiento y análisis de los procesos y resultados educativos y las innovaciones educativas no suelen acompañarse de un análisis de su impacto real en la práctica. En cualquier caso, no se aprovecha el conocimiento derivado de la investigación educativa para orientar la toma de decisiones en la materia, a pesar de la repetida retórica asociada con algunos discursos políticos. Este autor señala que el patrón generalizado es que los mundos de la política, la práctica y la investigación educativa están significativamente separados. Asimismo, Van den Akker (2013) afirma que establecer puentes constructivos entre estos tres ámbitos es una vía poderosa para aumentar las probabilidades de que las innovaciones curriculares redunden en la mejora de los procesos de enseñanza aprendizaje en las escuelas. Además, aun en los casos en los que la investigación se pone al servicio de la toma de decisiones políticas en temas de educación, con frecuencia se recurre a ella para generar estadísticas, análisis descriptivos o evaluaciones con el fin de informar el estado de la cuestión, pero son escasos los enfoques dirigidos a comprender las situaciones y hallar criterios que faciliten orientar el cambio y la mejora educativa. 


\section{Agradecimientos}

Se agradece en especial a las doctoras Nieveen y Pepin por la colaboración con la autora en la coordinación de la Escuela Internacional sobre DBR, celebrada en la Universidad de Jaén (España) en noviembre de 2013, así como por la publicación de los manuales sobre Educational Design Research editados por Nieveen y Plomp en el mismo año, que han constituido una auténtica fuente de inspiración y referencia para este trabajo.

\section{Sobre la autora}

Marta Romero-Ariza es doctora en Química Orgánica. Se desempeña como profesora de Didáctica de las Ciencias y vicedecana de Educación en la Universidad de Jaén. Participa en proyectos internacionales de investigación en formación de profesorado en Ciencias y Matemáticas (Compass, Primas, MaScil y Parrise), con especial interés en la utilización del IBL y el DBR como instrumentos de mejora.

\section{Referencias}

Bielaczyc, K., \& Ow, J. (2014). Multi-Player Epistemic Games: Guiding the Enactment of Classroom Knowledge-Building Communities. International Journal of Computer Based Collaborative Learning, 9(1), 33-62.

Bybee, R. W., Taylor, J. A., Gardner, A., Van Scotter, P., Powell, J. C., Westbrook, A., \& Landes, N. (2006). The BSCS 5E Instructional Model: Origins and Effectiveness. Recuperado de http://bscs.org/sites/default/files/_legacy/BSCS_5E_Instructional_Model-Executive_Summary_0.pdf

Cobb, P., Confrey, J., DiSessa, A., Lehrer, R., \& Schauble, L. (2003). Design Experiments in Educational Research. Educational Researcher, $32(1), 9-13$.

Cronbach, L. J. (1975). Beyond the Two Disciplines of Scientific Psychology. American Psychologist, 30(2), 116-127.

Dowse, C., \& Howie, S. (2013). Promoting Academic Research Writing with South African Master's Students in the Field of Education. En Plomp, T., \& Nieveen, N. (eds.). Educational Design Research. Enschede: SLO.

Duran, E., Duran, L., Haney, J., \& Scheuermann, A. (2011). A Learning Cycle for All Students. The Science Teacher, 78(3), 56-60.

Edelson, D. C. (2006). Balancing Innovation and Risk: Assessing Design Research Proposals. En Van den Akker, J., Gravemeijer, K., McKenney, S., \& Nieveen, N. (eds.). Educational Design Research. Londres: Routledge.

Eisenkraft, A. (2003). Expanding the 5E Model. The Science Teacher, 70(6), 56-59.

Fletcher, S. (2011). The Impact of the 6E Model on a Third Grade Science Classroom. (Tesis de maestría inédita). Bowling Green State University, Ohio, Estados Unidos.

Gravemeijer, K., \& Cobb, P. (2013). Design Research from the Learning Design Perspective. En Plomp, T., \& Nieveen, N. (eds.). Educational Design Research. Enschede: SLO.

Juuti, K., \& Lavonen, J. (2013). Design of a Primary School Physics WebBased Learning Environment: The Teacher's Role in the Educational Design Research Project. En Plomp, T., \& Nieveen, N. (eds.). Educational Design Research. Enschede: SLO. 
Kelly, A. E. (2006). Quality Criteria for Design Research: Evidence and Commitments. En Van den Akker, J., Gravemeijer, K., McKenney, S., \& Nieveen, N. (eds.). Educational Design Research. Londres: Routledge.

Knippels, M. C. P. J. (2002). Coping with the Abstract and Complex Nature of Genetics in Biology Education. (Tesis doctoral inédita). University of Utrecht, Utrecht, Países Bajos.

Krathwohl, D. R. (1998). Methods of Educational and Social Science Research: An Integrated Approach (2a ed.). Nueva York: Longman.

Mafumiko, F., Voogt, J., \& Van den Akker, J. (2013). Design and Evaluation of Micro-Scale Chemistry Experimentation in Tanzanian Schools. En Plomp, T., \& Nieveen, N. (eds.). Educational Design Research. Enschede: SLO.

McKenney, S. (2001). Computer-Based Support for Science Education Materials Developers in Africa: Exploring Potentials. (Tesis doctoral inédita). University of Twente, Enschede, Países Bajos.

McKenney, S., Nieveen, N., \& Van den Akker, J. (2006). Design Research from a Curriculum Perspective. En Van den Akker, J., Gravemeijer, K., McKenney, S., \& Nieveen, N. (eds.). Educational Design Research. Londres: Routledge.

Nieveen, N. (1999). Prototyping to Reach Product Quality. En Van den Akker, J., Branch, R. M., Gustafson, K., Nieveen, N., \& Plomp, T. (eds.). Design Approaches and Tools in Education and Training. Boston: Kluwer Academic.

Nieveen, N., \& Folmer, E. (2013). Formative Evaluation in Educational Design. En Plomp, T., \& Nieveen, N. (eds.). Educational Design Research. Enschede: SLO.

Plomp, T. (2013). Educational Design Research: An Introduction. En Plomp, T., \& Nieveen, N. (eds.). Educational Design Research. Enschede: SLO.

Reeves, T. C. (2000). Enhancing the Worth of Instructional Technology Research through "Design Experiments" and other Developmental Strategies. Recuperado de http://it.coe.uga.edu/ - treeves/AERA2000Reeves.pdf

Reeves, T. C. (2006). Design Research from a Technology Perspective. En Van den Akker, J., Gravemeijer, K., McKenney, S., \& Nieveen, N. (eds.). Educational Design Research. Londres: Routledge.

Siribuannam, R., \& Tayraukham, S. (2009). Effects of 7E, KWL, and Conventional Instruction on Analytical Thinking, Learning Achievement, and Attitudes toward Chemistry Learning. Journal of Social Sciences, 5(4), 279-282.

The Design-Based Research Collective. (2003). Design-Based Research: An Emerging Paradigm for Educational Inquiry. Educational Researcher, 32(1), 5-8.

Van den Akker, J. (1999). Principles and Methods of Development Research. En Van den Akker, J., Branch, R. M., Gustafson, K., Nieveen, N., \& Plomp, T. (eds.). Design Approaches and Tools in Education and Training. Boston: Kluwer Academic.

Van den Akker, J. (2005). Hoe kan samenwerking leiden tot meer succes en wijsheid in onderwijsontwikkeling?. Pedagogische Studiën, 82(4), 343-347.

Van den Akker, J. (2013). Curricular Development Research as a Specimen of Educational Design Research. En Plomp, T., \& Nieveen, N. (eds.). Educational Design Research. Enschede: SLO.

Van den Akker, J., Gravemeijer, K., McKenney, S., \& Nieveen, N. (eds.). (2006). Educational Design Research. Londres: Routledge. 
Wademan, M. R. (2005). Utilizing Development Research to Guide PeopleCapability Maturity Model Adoption Considerations. Recuperado de http://surface.syr.edu/idde_etd/12/

Yin, R. K. (2003). Case Study Research: Design and Methods. Newbury Park: SAGE.

Zuiker, S., \& Whitaker, R. (2014). Refining Inquiry with Multi-Form Assessment: Formative and Summative Assessment Functions for Flexible Inquiry. Journal of Science Education, 36(6), 1037-1059. 